\title{
Aerosol Optical Depth from MODIS satellite data above the Pierre Auger Observatory
}

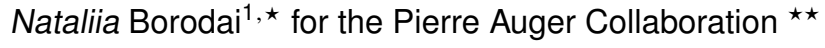 \\ ${ }^{1}$ Institute of Nuclear Physics Polish Academy of Sciences, Krakow, Poland
}

\begin{abstract}
Aerosol optical depth can be retrieved from measurements performed by Moderate Resolution Imaging Spectroradiometer (MODIS) satellite instrument. The MODIS satellite system includes two polar satellites, Terra and Aqua. Each of them flies over the Pierre Auger Observatory once a day, providing two measurements of aerosols per day and covering the whole area of the Observatory. MODIS aerosol data products have been generated by three dedicated algorithms over bright and dark land and over ocean surface. We choose the Deep Blue algorithm data to investigate the distribution of aerosols over the Observatory, as this algorithm is the most appropriate one for semi-arid land of the Pierre Auger Observatory. This data algorithm allows us to obtain aerosol optical depth values for the investigated region, and to build cloud-free aerosol maps with a horizontal resolution $0.1^{\circ} \times 0.1^{\circ}$. Since a sufficient number of measurements was obtained only for Loma Amarilla and Coihueco fluorescence detector (FD) sites of the Pierre Auger Observatory, a more detailed analysis of aerosol distributions is provided for these sites. Aerosols over these FD sites are generally distributed in a similar way each year, but some anomalies are also observed. These anomalies in aerosol distributions appear mainly due to some transient events, such as volcanic ash clouds, fires etc. We conclude that the Deep Blue MODIS algorithm provides more realistic aerosol optical depth values than other available algorithms.
\end{abstract}

\section{MODIS instruments and algorithms}

Terra and Aqua satellites with MODIS instruments attached fly on the sun-synchronous orbits and pass over the same spot of the Earth at about the same local time every day. Due to the large swath of data collected by MODIS (over $2300 \mathrm{~km}$ wide) it is possible to observe almost the entire Earth surface every day. The total area of the Pierre Auger Observatory [1] can be observed by one satellite during 1 orbit period or during 2 orbits, if divided between two swaths. MODIS measures reflected solar and emitted thermal radiation in a total of 36 bands [2], [3] with wavelengths between $0.41 \mu \mathrm{m}$ and $14.4 \mu \mathrm{m}$ for the entire MODIS field of view.

MODIS level 2 aerosol data products are used in this work. They are generated by the MODIS team from level 1B calibrated radiance data measured by the MODIS instruments. The full version of these data products is available in the collection 6 (C6) [3].

The level 1B MODIS data include measurements of so-called "sensor pixels" [4]. These are single measured pixels with the size $250 \mathrm{~m}, 500 \mathrm{~m}$, or $1 \mathrm{~km}$ at nadir, depending on the measured wavelength band. To decrease noise in the retrieval, adjacent sensor pixels are arranged to the larger blocks creating a second level scan, called "retrieval pixel". So, the level 2 aerosol products include only retrieval pixels. Each retrieval pixel has the size $10 \mathrm{~km} \times 10 \mathrm{~km}$ at nadir (that is approximately $0.1^{\circ} \times 0.1^{\circ}$ ).

\footnotetext{
${ }^{\star}$ e-mail: nataliia.borodai@ifj.edu.pl

${ }^{\star}$ Full author list: http://www.auger.org/archive/authors_2018_09.html
}

Only suitable (cloud and snow/ice-free) sensor pixels are taken to data retrieval during the retrieval procedure. The aerosol optical depth (AOD) value of a retrieval pixel is the average of the AOD values of the suitable sensor pixels inside this retrieval pixel.

There are two MODIS-over-land algorithms: the Dark Target (DT) algorithm [3] the Enhanced Deep Blue (DB) algorithm [2]. The DT algorithm was developed to retrieve AOD over dense, dark vegetation surfaces. The DB algorithm was used to retrive AOD over brighter arid, semiarid surfaces (such as deserts) and now it also covers vegetated land surfaces. Since the Pierre Auger Observatory is located in a semi-arid area, which is a bright surface type, the focus of this research is on the DB over-land data.

\section{Analysis procedure}

Each retrieval pixel is described in the DB database by the pixel center coordinates (longitude and latitude) and the AOD value (Deep_Blue_Aerosol_Optical_Depth_550_Land). To create the aerosol grid map, all the retrieval pixels are mapped to $0.1^{\circ} \times 0.1^{\circ}$ grid cells according to the positions of their centers. After that, the AOD values collected from both satellites during some period of time (day, month, year) are averaged in the cells and the aerosol map is plotted as a $2 \mathrm{D}$ histogram.

To fill the aerosol map with appropriate AOD values, the data selection procedure of MODIS AOD data was done. The data at the wavelength $550 \mathrm{~nm}$ 


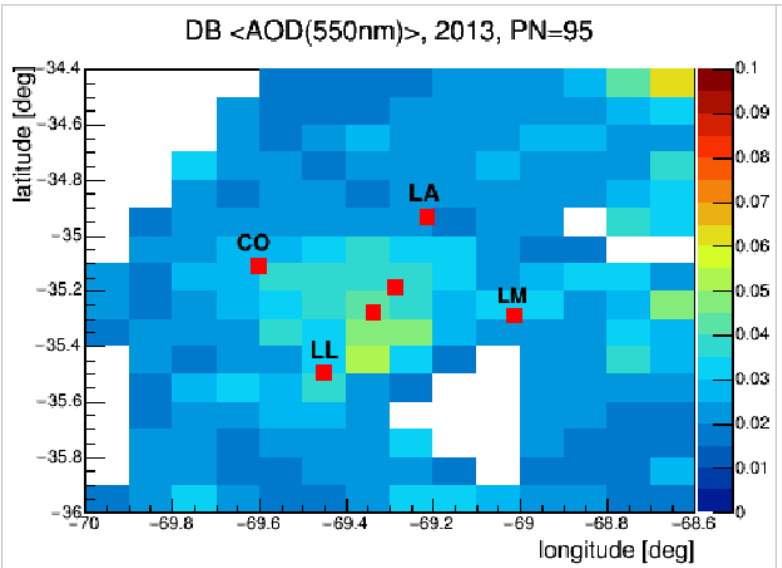

(a)

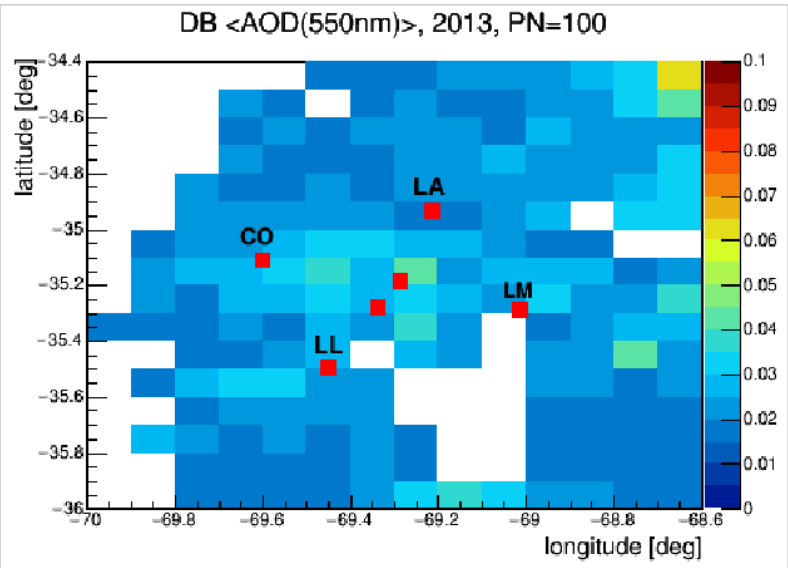

(b)

Figure 1. The Deep Blue <AOD> maps built for the year 2013 using MODIS AOD data at $550 \mathrm{~nm}$ from both satellites (Terra and Aqua). The maps differ by the PN cut used. Red squares correspond to the locations of the fluorescence detectors (FD) [1] Los Leones (LL), Los Morados (LM), Loma Amarilla (LA) and Coihueco (CO) and the laser facilities (CLF and XLF - two squares in the center).

are used in this work, as these are the only data with uncertainty available in the Deep Blue dataset. Data of the best quality are taken to this analysis (Deep_Blue_Aerosol_Optical_Depth_550_Land_QA_Flag, very good $=3$ ).

Only cloud-free sensor pixels are used in the MODIS data retrieval. To estimate the cloud fraction in a single retrieval pixel the parameter representing the number of suitable (cloud-free, snow-free) sensor pixels (Deep_Blue_Number_Pixels_Used_550_Land) is used. To reference this parameter in the following text, an abbreviation $\mathrm{PN}$ is used for number of pixels. So, the parameter PN shows how many cloud-free sensor pixels are in a retrieval pixel.

To estimate the PN, which is the optimal one to consider the retrieval pixel to be good for the analysis, we built the aerosol maps for $\mathrm{PN}=95, \mathrm{PN}=100$ (see Figure 1) for the Pierre Auger Observatory. As each retrieval pixel consists of 100 sensor pixels, the maximum for parameter PN is 100 and means that all sensor pixels inside the retrieval pixel are cloud-free. On these maps PN is a minimal number of suitable sensor pixels, which should be present inside a retrieval pixel, so that we can take this retrieval pixel to the analysis. So, if $\mathrm{PN}=95$, all retrieval pixels with 95 or more suitable sensor pixels inside are qualified to be used in the further analysis.

Only the PN criterion is changing in the aerosol maps in Figure 1(a) and (b), and even a small change of it causes the changes of the $<\mathrm{AOD}>$ values on maps. By changing the PN criterion from 95 to 100 we also decrease the number of measurements in each cell. So, the map with the numbers of measurements corresponding to aerosol map in Figure 1(b) with $\mathrm{PN}=100$ is shown in Figure 2.

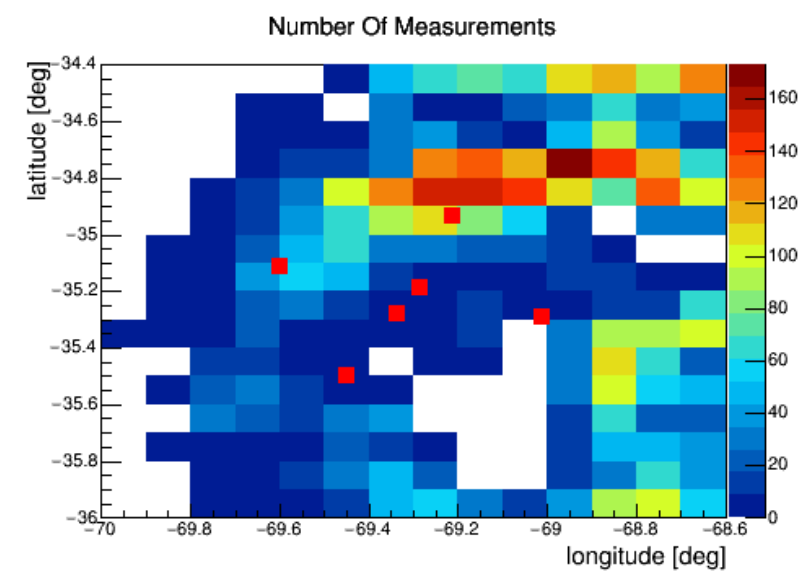

Figure 2. The map with the numbers of measurements corresponding to Figure 1(b) the Deep Blue aerosol map.

The dependence of $<$ AOD $>$ for June and November 2013 and number of measurements on PN values is also shown in a Table 1 . For $\mathrm{PN}=50$ the number of measurements is large, but the statistical uncertainty of $<\mathrm{AOD}>$ values is large as well. When $\mathrm{PN}=100$, the number of measurements decreases significantly, but $<\mathrm{AOD}>$ values are much lower than with smaller PN. So, when we have some fraction of cloudy sensor pixels inside a retrieval pixel, it affects much AOD estimation inside this retrieval pixel, leading finally to unreasonably high $<\mathrm{AOD}>$ of "cloudfree" retrieval pixel. Consequently, the best way to minimize the cloud effect on "cloud-free" pixels is to select pixels with $\mathrm{PN}=100$, i.e. all sensor pixels are cloud-free.

One more selection criterion is the choice of the right algorithm. As it was already mentioned in section 1, MODIS data processed using DB algorithm should be

Table 1. The $<$ AOD $>$ for June and November 2013 depending on suitable number of sensor pixels (PN) per retrieval pixel. Statistical errors are calculated. The number of measurements decreases with increasing requirement on the number PN.

\begin{tabular}{|l|l|l|l|}
\hline $\begin{array}{l}\text { Number of Sensor Pixels } \mathbf{( P N )} \\
\text { per Retrieval Pixel }\end{array}$ & $\begin{array}{l}\text { Total number of } \\
\text { measurements in year 2013 }\end{array}$ & <AOD> in June & <AOD> in November \\
\hline over 50 pixels & 28719 & $0.0315+/-0.1411$ & $0.0306+/-0.0404$ \\
\hline over 81 pixels & 22549 & $0.0255+/-0.0833$ & $0.0291+/-0.0252$ \\
\hline over 95 pixels & 14270 & $0.0225+/-0.0395$ & $0.0287+/-0.0262$ \\
\hline 100 pixels & 8740 & $0.0205+/-0.0075$ & $0.0282+/-0.0298$ \\
\hline
\end{tabular}




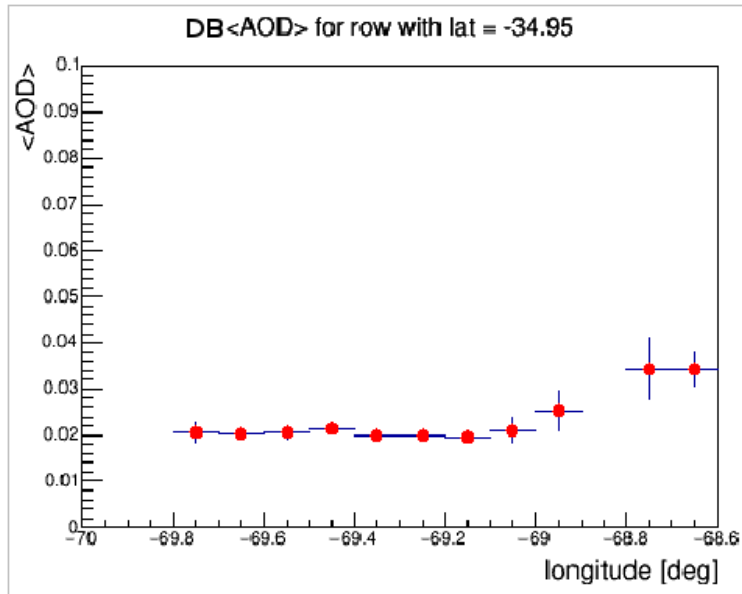

(a1)

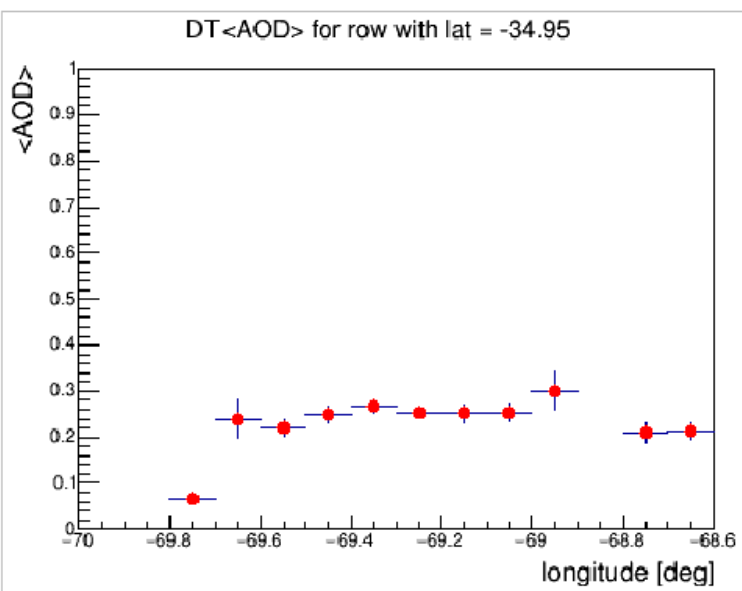

(b1)
$D B<A O D>$ for row with lat $=-35.15$

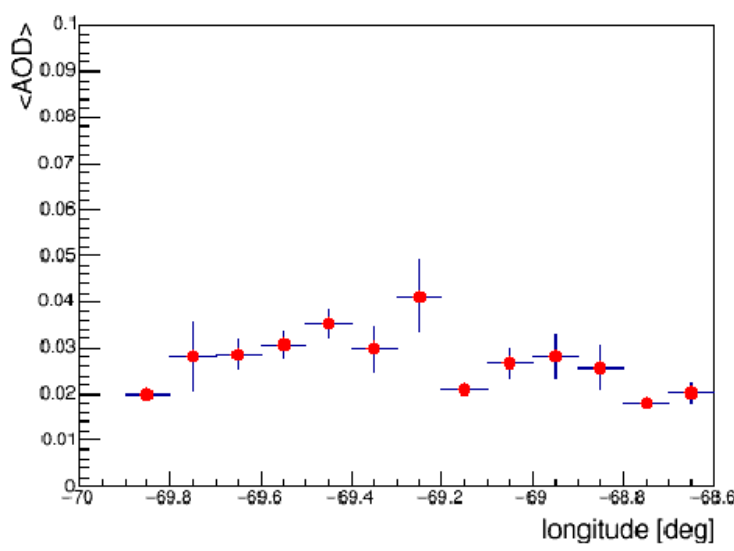

(a2)

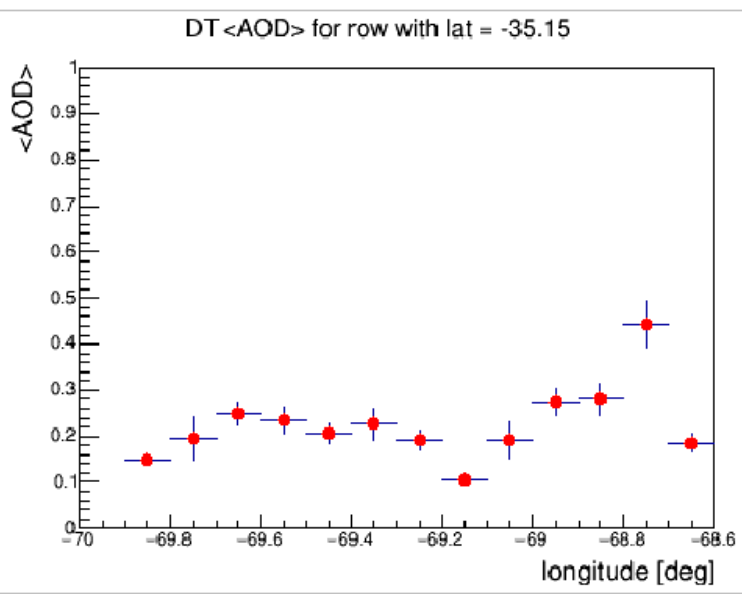

(b2)

Figure 3. (a1) and (a2) - the East-West $<$ AOD $>$ profiles of the DB aerosol map (from Figure 1(b)) for $550 \mathrm{~nm}$ for the year 2013 with the values of $<\mathrm{AOD}>$ corresponding to the rows with latitudes $-34.95 \mathrm{deg}$ and $-35.15 \mathrm{deg}$. (b1) and (b2) are the profiles for the same coordinates and time moment as two previous plots, but plotted using the Dark Target data. <AOD $>$ for DT profiles (b1,b2) is around 0.2 that is 10 times higher than obtained using the Deep Blue algorithm (a1,a2).

used for the Pierre Auger Observatory. If the other (DT) algorithm is used, the $<\mathrm{AOD}>$ values become very large and do not reflect the real aerosol distribution. For comparison the East-West $<$ AOD $>$ profiles of the DB aerosol map (from Figure 1(b)) and profiles of the DT aerosol map are shown in Figure 3. They present values of $<$ AOD $>$ from two rows of aerosol map. These rows with latitudes $-34.95 \mathrm{deg}$ and $-35.15 \mathrm{deg}$ include the latitudes of LA and $\mathrm{CO}$ detectors. If we compare the values of DT $<\mathrm{AOD}>$ with the same ones obtained using the DB algorithm, it is

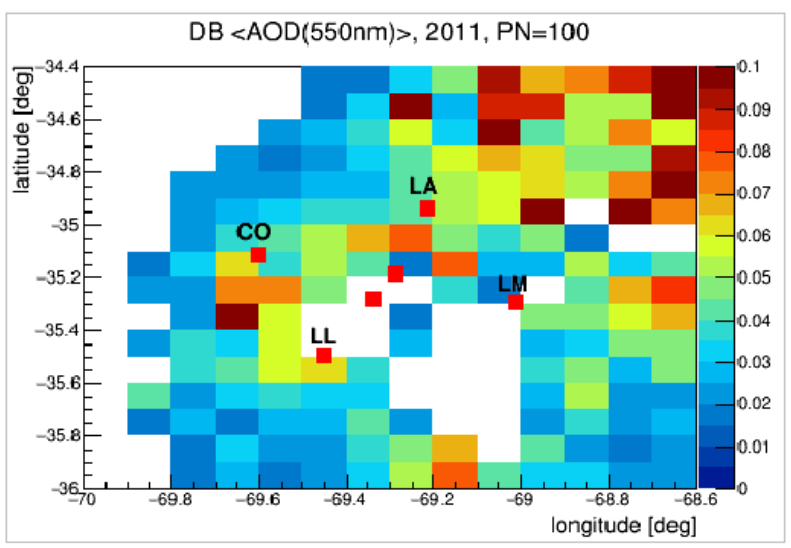

Figure 4. DB <AOD> map for $550 \mathrm{~nm}$ for the year 2011. obvious that DT values are overestimated as they are 10 times higher than obtained using the Deep Blue algorithm.

There are two large empty regions with no data on maps shown in Figure 1. One is between LL and LM detectors. It corresponds to the Llancanelo Lake and so cannot be retrieved by DB or DT over land algorithms as it is not land. The second empty region is on the left-hand side of the maps. It corresponds to snowy Andes Mountains and so cannot be retrieved by the discussed algorithms.

\section{Interpretation of maps and daily plots}

Figure 1(b) presents the data which satisfy the selection criteria imposed on MODIS AOD data. Using aerosol maps it is possible to distinguish years with clear atmosphere above the Pierre Auger Observatory and years with some anomalies in aerosol distribution. For example, Figure 4 demonstrates the aerosol map prepared for the year 2011. By comparing this map with the one from the year 2013 (Figure 1(b)), it can be concluded that in 2013 the aerosol distribution was uniform and the atmosphere was clear, while in 2011 some anomalies appeared above the Pierre Auger Observatory as many increased values of $<$ AOD $>$ appeared on the map.

Daily plots are also useful to distinguish the years with anomalies. Same-day $<$ AOD $>$ measurements, which sat- 


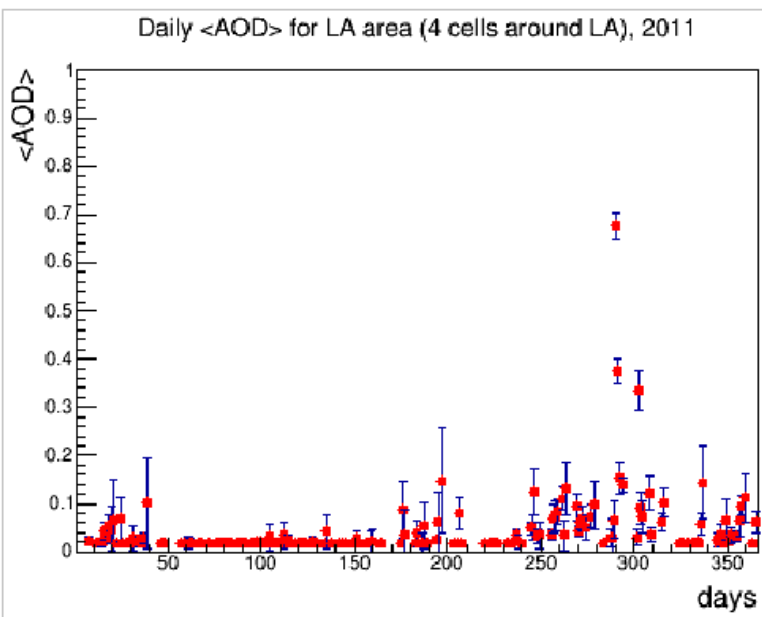

(a1)
Daily <AOD> for CO area (4 cells around CO), 2011

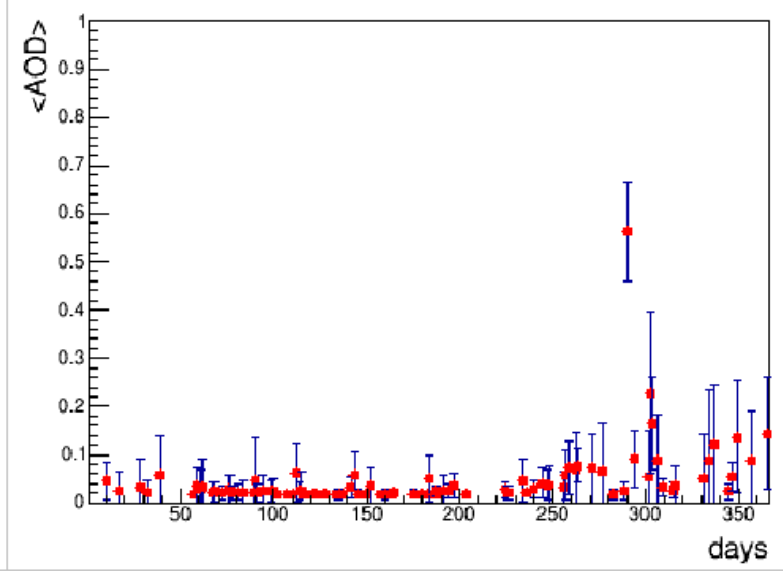

(a2)

Figure 5. Daily $<$ AOD $>$ plots for $550 \mathrm{~nm}$ for the year 2011 for LA (a1) and CO (a2). Weighted mean $<$ AOD $>$ values and uncertainty of weighted mean are shown. The increase of $\angle A O D>$ is observed in the plots as well as in the aerosol map for this year (Figure 4). Days scale is the day number in a year.

isfy the selection criteria, can be averaged to produce the daily $<$ AOD $>$ plots. As the DB dataset also provides an estimate of the uncertainty (one-standard-deviation confidence interval) on $<\mathrm{AOD}>$ at $550 \mathrm{~nm}$ for each retrieval pixel [5], weighted daily mean and uncertainty of weighted mean can be plotted. It can be concluded from Figure 2 that the number of measurements in the areas of LA and CO FD sites of the Pierre Auger Observatory is much higher than in other FD locations. So, we prepared the daily $<\mathrm{AOD}>$ plots for LA and CO sites. For this purpose four grid cells closest to each FD site are chosen. The $<$ AOD $>$ measurements from these cells are averaged for each site and daily $<$ AOD $>$ plots are produced.

Daily plots for LA and CO FD sites for the year 2011 (see Figure 5) include some period of time with increased daily $<\mathrm{AOD}>$ up to the peak at 0.7 for LA and at 0.6 for $\mathrm{CO}$. So, daily $<\mathrm{AOD}>$ data for both sites give similar plots with maximum about days 290-291 of measurements. These days correspond to 17-18 October 2011 and are within the period (14-18 October 2011) when volcanic ash resuspension and dispersal by the strong $(\approx 100 \mathrm{~km} / \mathrm{h})$ wind happened in the large part of Argentina [6]. During that period of time, a widespread ash cloud was formed at the altitude about $1.5 \mathrm{~km}$. This cloud dispersed rapidly over large part of Argentina, including Córdoba, Mendoza and San Luis provinces making the sky almost invisible.

Taking into account this information, it can be concluded that the increase of daily $\langle\mathrm{AOD}\rangle$, which started in Figure 5(a1) after 170th day of a year (corresponding to 19 June 2011) and continued up to the end of the year, can be caused by appearing of clouds with volcanic ash in the atmosphere above the Pierre Auger Observatory, taken there by the strong winds.

\section{Summary}

The MODIS Terra and Aqua datasets were analyzed in this study. A comparison of AOD values obtained using the Dark Target and Deep Blue algorithms shows that they differ much. The reason for this difference is the bright land surface of the Pierre Auger Observatory for which the DB algorithm (and not DT) should be used.

The MODIS algorithms are still under development. Although the implementation of the DB algorithm improved much the AOD data obtained over the Pierre Auger Observatory, it is still difficult to make a good comparison of MODIS data with the Auger CLF data, because of the quality of MODIS data. Therefore, the MODIS algorithms should be considerably improved. Nevertheless, the present MODIS satellite data can probably be used for detection of some global anomalous distributions of aerosols over the Pierre Auger Observatory for the purpose of the subsequent tracing these anomalies in the Auger aerosol data.

\section{Acknowledgments}

The MODIS/Terra and the MODIS/Aqua Aerosol 5Min L2 Swath 10km (MOD04_L2 and MYD04_L2) datasets were acquired from the Level-1 and Atmosphere Archive \& Distribution System (LAADS) Distributed Active Archive Center (DAAC), located in the Goddard Space Flight Center in Greenbelt, Maryland (https://ladsweb.nascom.nasa.gov/).

This work was partially supported by the National Science Centre, grant NO. 2016/23/B/ST9/01635.

\section{References}

[1] The Pierre Auger Collaboration, Nucl. Instr. and Meth. A 798, 172-213 (2015).

[2] N. C. Hsu et al., J. Geophys. Res. Atmos. 118, 9296-9315 (2013).

[3] R. C. Levy et al., Atmos. Meas. Tech. 6, 2989-3034 (2013).

[4] A. M. Sayer et al., Atmos. Meas. Tech. 8, 5277-5288 (2015).

[5] A. M. Sayer et al., J. Geophys. Res. Atmos. 118, 7864-7872 (2013).

[6] A. Folch et al., Nat. Hazards Earth Syst. Sci. 14, 119-133 (2014). 